\title{
Computational discovery and RT-PCR validation of novel Burkholderia conserved and Burkholderia pseudomallei unique sRNAs
}

\author{
Jia-Shiun Khoo ${ }^{1,2}$, Shiao-Fei Chai ${ }^{1}$, Rahmah Mohamed ${ }^{1}$, Sheila Nathan ${ }^{1,3}$, Mohd Firdaus-Raih ${ }^{1 *}$ \\ From Asia Pacific Bioinformatics Network (APBioNet) Eleventh International Conference on Bioinformatics \\ (InCoB2012) \\ Bangkok, Thailand. 3-5 October 2012
}

\begin{abstract}
Background: The sRNAs of bacterial pathogens are known to be involved in various cellular roles including environmental adaptation as well as regulation of virulence and pathogenicity. It is expected that sRNAs may also have similar functions for Burkholderia pseudomallei, a soil bacterium that can adapt to diverse environmental conditions, which causes the disease melioidosis and is also able to infect a wide variety of hosts.

Results: By integrating several proven sRNA prediction programs into a computational pipeline, available Burkholderia spp. genomes were screened to identify sRNA gene candidates. Orthologous SRNA candidates were then identified via comparative analysis. From the total prediction, 21 candidates were found to have Rfam homologs. RT-PCR and sequencing of candidate sRNA genes of unknown functions revealed six putative sRNAs which were highly conserved in Burkholderia spp. and two that were unique to B. pseudomallei present in a normal culture conditions transcriptome. The validated sRNAs include potential cis-acting elements associated with the modulation of methionine metabolism and one B. pseudomallei-specific sRNA that is expected to bind to the Hfq protein.

Conclusions: The use of the pipeline developed in this study and subsequent comparative analysis have successfully aided in the discovery and shortlisting of sRNA gene candidates for validation. This integrated approach identified 29 B. pseudomallei sRNA genes - of which 21 have Rfam homologs and 8 are novel.
\end{abstract}

\section{Introduction}

Small RNAs (sRNAs) are known to function as regulatory or catalytic molecules in bacteria with sequences normally ranging from $\sim 50-250 \mathrm{nt}$ in length and located in the intergenic regions (IGRs) [1,2]. Although sRNAs with catalytic functions have been reported [3,4], many of these molecules are known or believed to function as regulatory nucleic acid elements that target near, or at, the translation start site of their dedicated mRNA targets via imperfect sequence complementarity [5-7]. In E. coli, less than 100 sRNAs, accounting for $\sim 0.3 \%$ of the genome, have

\footnotetext{
* Correspondence: firdaus@mfrlab.org

'School of Biosciences and Biotechnology, Faculty of Science and

Technology, Universiti Kebangsaan Malaysia, 43600 UKM Bangi, Malaysia Full list of author information is available at the end of the article
}

been reported [8-10]. Although these riboregulators represent only a small fraction of the prokaryotic genome, they have been shown to play essential regulatory roles in bacteria, including cell surface modulation [11], plasmid number control [12], stress adaptation [13], quorum sensing [14] and carbon storage [15]. Other regulatory sRNAs interact with and modulate cellular protein activities [16].

In pathogenic bacteria, sRNAs have been associated with regulatory networks that modulate the adherence to, and invasion into the host cell $[17,18]$, environmental adaptation $[19,20]$ as well as virulence and pathogenicity [17,18,20-23]. In several bacterial pathogens, including Salmonella typhimurium [24], Vibrio cholerae [25], Yersinia enterocolitica [26], Brucella abortus [23] and Pseudomonas aeruginosa [27], deletion of the $h f q$ gene

C Biomed Central

(c) 2012 Khoo et al.; licensee BioMed Central Ltd. This is an open access article distributed under the terms of the Creative Commons Attribution License (http://creativecommons.org/licenses/by/2.0), which permits unrestricted use, distribution, and reproduction in any medium, provided the original work is properly cited. 
which encodes the RNA chaperone Hfq, has been shown to severely attenuate virulence. The Hfq protein is known to facilitate the pairing interaction between sRNAs and their target mRNAs [28]. Identification and analysis of sRNAs in pathogenic bacteria may improve current understanding on the molecular mechanisms of host adaptation and virulence. Hence, we carried out a computational based analysis of available Burkholderia spp. genomes to identify potential sRNA sequences and to further delineate sRNAs that are present only in the pathogenic members.

Members of the Burkholderia genus also play important roles as environmental saprophytes. One species of this genus, $B$. pseudomallei, is the causative agent of melioidosis, a disease endemic to Southeast Asia and northern Australia. This species reportedly has a highly dynamic genome and versatile phenotypes [29-31], thus contributing to its capability to infect nearly all cell types, resulting in a wide spectrum of disease symptoms that confounds diagnosis and delays prompt treatment. B. pseudomallei is an effective pathogen of a broad range of hosts (amoeba [32], nematodes [33], dolphins [34], birds, camels, alpacas, sheep [35], humans and even plants [36]). The enigma of $B$. pseudomallei is further compounded in having an extremely prolonged latent infection capacity [36] and has been shown to be capable of surviving in a nutrient-free environment for 16 years [37].

B. pseudomallei is believed to have an array of virulence and pathogenicity factors, including a toxin which is a deamidase named Burkholderia Lethal Factor 1 (BLF1) that targets the translation initiation factor eIF4a [38]. However, the regulation and delivery mechanism of BLF1 to the target protein remains unclear. To date, the mechanisms of adaptation to environmental stress and changes have not been conclusively identified, however a large number of sRNA genes have been reported for B. cenocepacia $\mathrm{J} 2315$, another pathogenic member of the Burkholderia genus [39]. These sRNAs were proposed to be responsible for the bacterium's complexity, phenotypic variability and ability to survive in a remarkably wide range of environments [39].

At present, one can opt for either a knowledge-based approach or a de novo approach for sRNA discovery in a bacterial genome. Knowledge-based techniques search for homologues of known sRNAs based on specific features of the sequences and will usually include upstream regulatory elements, sequence and structural characteristics and downstream targets as a search profile. A number of knowledge-based programs were developed to identify particular sets of sRNAs through homology analysis. One such program, Infernal [40], was the workhorse used to build the Rfam database [9]. However, predictions relying on homology information limit the applications of such programs to sRNA genes with known homologues and therefore, the methods are insufficient in situations where many if not most bacterial sRNAs remain unidentified. A de novo approach can serve a complementary role in predicting novel sRNA genes that are beyond the profile scope of knowledge-based approaches. The basis of a de novo search lies in the common features of sRNAs in the genomes - sequence and structural conservation, shared physical co-localization, structural stability, existence of transcriptional signals and GC bias - without prior knowledge of the sRNAs to be discovered. Such an approach was applied with various sRNA gene finders such as QRNA [41], RNAz [42,43], sRNAPredict [44,45] and sRNAscanner [46]. In this paper, we report the development of a computational pipeline that integrated successful sRNA prediction programs to identify candidate sRNA genes in B. pseudomallei and subsequent validation by RT-PCR and Sanger sequencing.

\section{Methods}

\section{Development of the sRNA gene detection pipeline}

A computational pipeline for bacterial sRNA gene prediction was developed by integrating the output of three published sRNA detection programs; Rfam_scan [9], SIPHT [48] and sRNAscanner [46]. The pipeline consists of a number of core programs for data format conversion and consensus identification and a main program (GetsRNA. $\mathrm{pl}$ ) that controls the data flow between the elementary scripts (Figure 1). sRNA candidates were then named according to the following convention $\mathrm{Bp}$ [Chromosome number]_[candidate number for program]_[program name] eg. Bp1_Cand612_SIPHT.

The intergenic sequences (here, defined as sequences between annotated ORFs) of the replicons were extracted using Artemis v12.0.3 [49] and searched against the Rfam database v10.0 by executing the script rfam_scan.pl v1.0. The supporting software used for the search included BLAST v2.2.22 [50], Infernal v1.0, Perl v5.10.0 and BioPerl v1.6.0.

SIPHT searches were restricted to detect sRNA genes within the range of 30-550 nucleotides and executed via the web server (URL: http://newbio.cs.wisc.edu/sRNA/). Other parameters were optimized as suggested [48]; i.e., maximum E value: $1 \mathrm{e}-15$, minimum TransTerm confidence value: 87 , maximum FindTerm score: -10, maximum RNAMotif score: -9 . All replicons, except the replicon of interest, were included as a partner replicon for the search.

The program sRNAscanner_Ubuntu10 (released 31 August 2010) was used to screen both the forward and reverse strands of the query replicon. The searches were restricted to intergenic regions and the sRNA length for prediction was set to 30-550 nucleotides. All other parameters were left at their default values, i.e. 3 provided input matrices: 35box_sRNA.matrix (cut-off: 2), 10box_sRNA. 


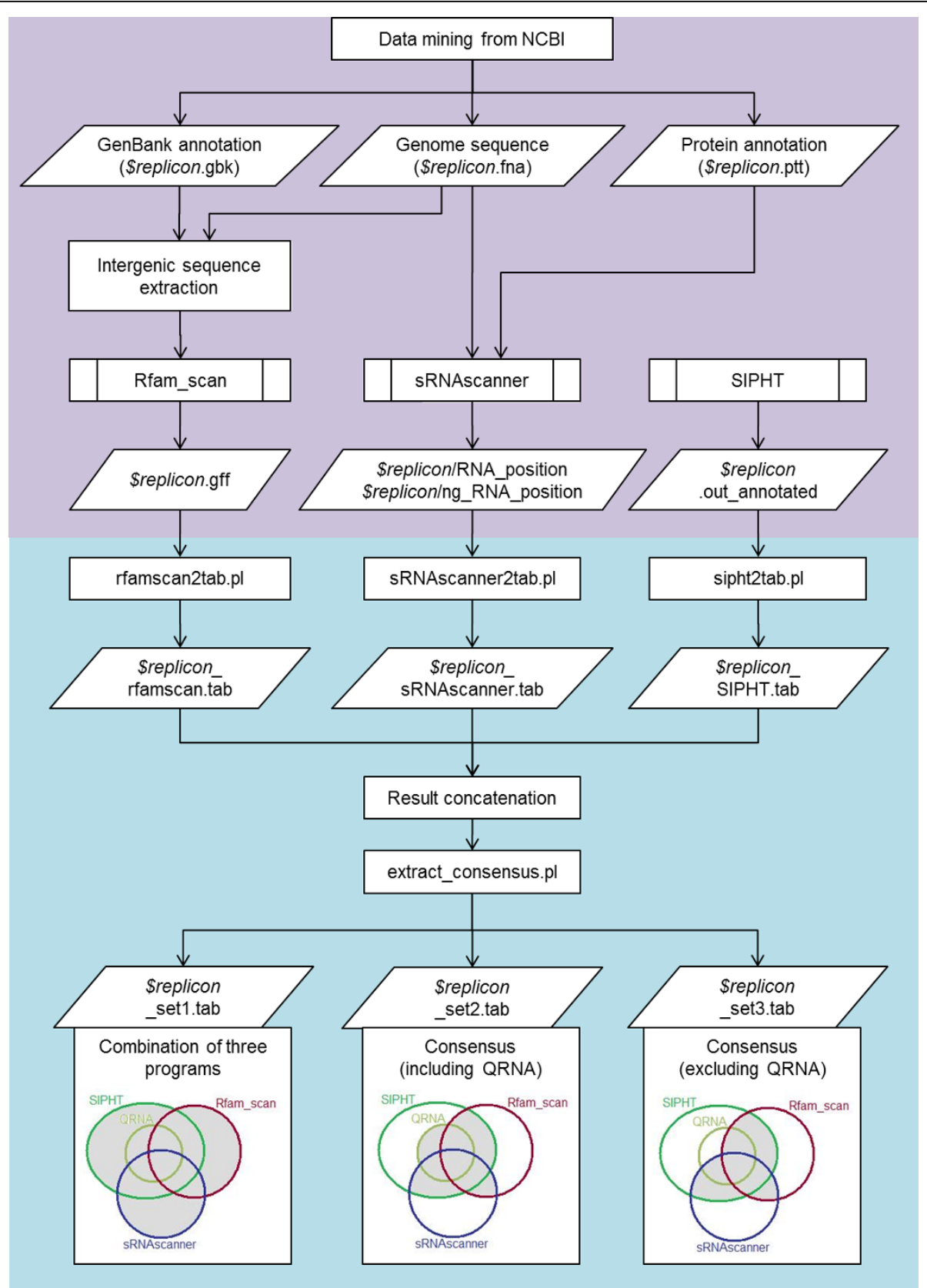

Figure 1 Pipeline for bacterial sRNA gene prediction. The steps in the purple zone (top) were executed manually while the steps in the blue zone (bottom) were automated using Perl script GetRNA.pl developed in this study. The variable sreplicon refers to the replicon tested.

matrix (cut-off: 2), terminator.txt.matrix (cut-off: 3); spacer range between [-35] \& [-10] promoter boxes: 12-18; unique hit value: 200; minimum cumulative sum of score (CSS): 14 .

\section{Genome sequences, annotation files and databases}

The genome sequences of 11 Burkholderia spp and 3 Ralstonia spp (.fna extensions), annotation files (.gbk and .ptt extensions) and the complete genomic sequences of RefSeq-release47 (.genomic.fna extensions) were obtained from NCBI (Additional file 1). The genome sequences of five local strains of $B$. pseudomallei (unpublished data) were used for cross-referencing purposes. The Rfam database v10.0, both .fasta and .cm extensions for 1,446 sRNA families, was downloaded from ftp://ftp.sanger.ac. uk/pub/databases/Rfam/.

\section{Comparative analysis}

The intergenic sequences of B. pseudomallei K96243 were compared to sRNA candidates predicted in the Ralstonia and Burkholderia genomes using blastn 
v2.2.21 (parameters: -e 1e-5 -r 1 -q -1 -G 1 -E 2 -W 9 $-F$ “m D"). The results were visualized using ACT v9.0.3 [51] and the gene physical co-localization for the sRNAs of interest were investigated.

\section{Secondary structure prediction}

The secondary structures of the sRNA transcripts were predicted using mfold (unafold v3.8) [52] and RNAfold (ViennaRNA v1.8.4) [53]. The default parameters or standard conditions for RNA folding were accepted $\left(37^{\circ} \mathrm{C}\right.$, $1 \mathrm{M} \mathrm{NaCl}$, no divalent ions). The predicted structures were visualized using VARNA v3.7 [54].

\section{Homologue detection}

Sequences for sRNAs of interest were globally aligned and consensus secondary structures were predicted using LocARNA [55] via its web service (URL: http://rna.tbi. univie.ac.at/cgi-bin/LocARNA.cgi). The default parameters for scoring the alignments were accepted (RIBOSUM85_60 matrix, Indel-opening score: -500, Indel score: -350 , structure weight: 180 , avoid lonely basepairs). Covariance models representing the alignments with consensus structures were built, calibrated and searched against complete genome sequences in the RefSeq database release 47 using Infernal v1.0 with an Evalue $\geq 1 \mathrm{e}-3$.

\section{B. pseudomallei strain and RNA extraction}

The B. pseudomallei D286 human isolate was obtained from the Pathogen Laboratory, School of Biosciences and Biotechnology, Faculty of Science and Technology, Universiti Kebangsaan Malaysia, Malaysia. Stock cultures were stored at $-70^{\circ} \mathrm{C}$ and routinely cultured on brain-heart infusion agar (BHIA) (Pronadisa Hispanlab, South Africa) at $37^{\circ} \mathrm{C}$ [56]. Bacteria from a stock culture were taken and streaked on Ashdown agar, and incubated at $37^{\circ} \mathrm{C}$ for 48 hours. A single colony was picked from the plate and inoculated into Brain Heart Infusion broth (BHIB) overnight. The following day, the culture was diluted 1:100 and grown in BHIB until the $\mathrm{OD}_{600}$ reached $0.6-1.0$. Total RNA was extracted using TRIzol ${ }^{\circledR}$ LS Reagent (Invitrogen, Carlsbad, CA) and purified using Ambion's DNAfree $^{\mathrm{TM}}$ DNase Treatment and Removal Reagents (Life Technologies, Carlsbad, CA).

\section{Reverse transcription polymerase chain reaction (RT-PCR) and Sanger sequencing}

The purified RNA was reverse transcribed into cDNA with an oligo(dT)18 primer using RevertAid First Strand cDNA Synthesis Kit (Fermentas, Hamburg, Germany). The cDNA produced was used as the template for PCR together with primers that were designed based on the sequences of sRNA candidates (Additional file 2). Amplification reactions were performed in a total volume of
$25 \mu \mathrm{L}$ consisting 10x PCR buffer, $10 \mathrm{mmol} / \mathrm{L}$ of $\mathrm{dNTP}$ mix, approximately $100 \mathrm{ng}$ of cDNA, $25 \mathrm{pmol}$ of each primer, 1.0 U Taq polymerase (Promega, Madison, WI) and distilled water. Mastercycler ${ }^{\circledR}$ personal (Eppendorf, Hamburg, Germany) was used to perform gradient PCR, with an initial denaturation step of 2 minutes at $95^{\circ} \mathrm{C}$, followed by 35 amplification cycles of 30 seconds at $95^{\circ} \mathrm{C}, 30$ seconds at $54-62^{\circ} \mathrm{C}$, and 30 seconds at $72^{\circ} \mathrm{C}$, and a final extension of 2 minutes at $72^{\circ} \mathrm{C}$. Amplified products were analyzed by $3 \%$ agarose gel electrophoresis with O'GeneRuler $^{\mathrm{TM}}$ Low Range DNA Ladder (Fermentas, Vilnius, Lithuania) run in parallel. PCR products were purified with the QIAquick Gel Purification Kit (Qiagen, Germany) and used in the reaction with the BigDye ${ }^{\circledR}$ Terminator v3.1 Cycle Sequencing Kit (Applied Biosystem, Foster City, CA). Three biological replicates were carried out for each RTPCR primer sets. The PCR products were then sequenced on the ABI Prism ${ }^{\circledR} 3100$ AVANT DNA Sequencer. The sequences obtained were analyzed using BioEdit v7.3.1.0 and compared with the genome sequence of B. pseudomallei D286 human isolate.

\section{Results and discussion}

\section{Pipeline development and performance assessment}

Several computational approaches for sRNA discovery have been used on various bacterial genomes to successfully identify and validate tens to hundreds of putative sRNA genes (Table 1). Due to resource limitations, it is common practice for only a limited number of the hundreds to thousands of computationally identified sRNAs to be selected for experimental verification and characterization. The percentage of validated sRNAs relative to the total number of predicted candidates can be as high as $37.6 \%$ as in the case for Streptomyces coelicolor [47] to as low as $0.1 \%$ in B. cenocepacia [39]. The percentage of verified sRNAs over the number of computationally predicted candidates which were tested is similarly varied; $78.7 \%$ for S. coelicolor and $1.88 \%$ for B. cenocepacia. In some cases, the verification experiments are inconclusive due to the uncertainty of whether a target transcript was transcribed under the particular experimental or culture conditions used. As a result, the number of sRNAs validated experimentally is usually smaller than the number of sRNAs originally selected for verification from the computationally predicted list (Table 1).

Three different sRNA predictors Rfam_scan [9], SIPHT [48] and sRNAscanner [46], each with different sets of criteria used to identify bacterial sRNAs, were selected to be the elementary programs in the sRNA prediction pipeline developed. The integrated sRNA prediction pipeline (Figure 1) enabled the discovery of sRNA genes in the target genome sequences of organisms from the order Burkholderiales via analysis of the consensus results. The performance of the pipeline was initially assessed using 
Table 1 Discovery and verification of bacterial sRNAs in previous studies.

\begin{tabular}{|c|c|c|c|c|c|c|}
\hline \multirow[t]{2}{*}{ Bacteria } & \multirow[t]{2}{*}{ Computational discovery method } & \multirow[t]{2}{*}{ Verification method } & \multicolumn{3}{|c|}{ Number of sRNAs } & \multirow[t]{2}{*}{ Reference } \\
\hline & & & Predicted & Tested & Verified & \\
\hline Escherichia coli & QRNA & Northern blot & 275 & 49 & 11 & {$[64]$} \\
\hline Escherichia coli & Pftools2.2 \& RNAMotif & Northern blot & 227 & 8 & 7 & {$[65]$} \\
\hline Burkholderia cenocepacia & QRNA & Microarray & 3,441 & 213 & 4 & {$[39]$} \\
\hline Streptomyces coelicolor & BLAST \& TransTermHP & RT-PCR \& microarray & 37 & 32 & 20 & {$[66]$} \\
\hline Synechocystis PCC6803 & RNAz & Northern blot & 383 & 2 & 2 & {$[67]$} \\
\hline Staphylococcus aureus & RNAsim & Northern blot & 774 & 36 & 11 & {$[68]$} \\
\hline Escherichia coli & Anonymous program & Northern blot & 601 & 6 & 3 & {$[69]$} \\
\hline Salmonella enterica Typhimurium & sRNAscanner & Northern blot & 156 & 16 & 6 & {$[46]$} \\
\hline Streptomyces coelicolor & RNAz \& nocoRNAc & Microarray & 843 & 403 & 317 & {$[47]$} \\
\hline Burkholderia pseudomallei & SIPHT, sRNAscanner \& Rfam_scan & RT-PCR & 1306 & 15 & 8 & This study. \\
\hline
\end{tabular}

the benchmark datasets and assessment method reported by Lu et al. [57]. The three sets of outputs from the pipeline, as well as predictions by individual elementary programs in the pipeline were evaluated using the ten sets of benchmark sRNAs (Figure 2). The highest mean sensitivity was achieved by compiling all the predictions (output set1) resulting in a retrieval rate of $48.88 \%$; the next most sensitive approach, with $30.65 \%$ retrieval, was output set2, i.e. an overlap of the different outputs integrated by our pipeline to derive a consensus predicted sRNA list (which included QRNA output). In the case of precision assessment, output set3, i.e. consensus predicted sRNA list (excluding QRNA output) outperformed other methods by achieving a mean precision of $29.90 \%$. By using the $F_{1}$ measure (which puts equal weight on sensitivity and precision) and $F_{0.25}$ measure (which puts the weightage on precision at four times more than sensitivity) as proxies of prediction accuracy [58]; it was revealed that output set3 from the pipeline achieved the highest mean performance, i.e., $17.35 \%$ and $25.26 \%$ respectively for each measure. In addition, output set 3 also outperformed the other prediction methods in predicting the extent of sRNAs by identifying $81.87 \%$ of sRNA nucleotides on average. However, the highest ability to correctly identify the transcription directions of these sRNAs was achieved by SIPHT, i.e., $86 \%$ of the time on average. The pipeline enabled us to carry out the computational annotation of sRNA genes for available Burkholderia genomes by analyzing the conservation of predicted candidates in different species from the genuses Burkholderia and Ralstonia. Additionally, by interrogating the genome sequences of different $B$. pseudomallei isolates, we were able to identify conserved sRNA candidates that are unique to B. pseudomallei and are discussed further below.

\section{SRNA searches in B. pseudomallei and other related species}

Fourteen genomes (Additional file 1) including B. pseudomalle $i$ were searched for sRNA genes using the pipeline developed. A total of 8,920 individual sRNA candidates were returned from the searches (output set1), with the smallest number, 193, for R. solanacearum, and the largest number, 1,306, for B. pseudomallei. Files containing the locations of all predicted sRNA genes are available as Additional file 3 . The 8,920 sRNA candidates identified, varied in length between 24 and $551 \mathrm{nt}$ (Figure 3A). The majority of the sRNA candidates (78.26\%) were 51-250 nt in length. The $\mathrm{G}+\mathrm{C}$ percentage of the sRNA candidates ranged from $25.44 \%$ to $89.15 \%$. The $\mathrm{G}+\mathrm{C}$ content distribution of sRNA candidates (Figure $3 \mathrm{~B}$ ) suggested that most of the sRNA candidates $(82.72 \%)$ have a $\mathrm{G}+\mathrm{C}$ content higher than $55 \%$. As sRNAs are diverse in both functions and mechanisms of action, various $\mathrm{G}+\mathrm{C}$ content in the sRNAs would be expected to fulfill different requirements of stability. From previous studies [8,59], the $\mathrm{G}+\mathrm{C}$ content of sRNAs were found to be higher than in the associated IGRs. However, we found that the overall $\mathrm{G}+\mathrm{C}$ content of sRNAs predicted for the 14 genomes in our work was generally comparable to the $\mathrm{G}+\mathrm{C}$ content of the IGRs (Figure $3 \mathrm{C}$, line graph). This could however be attributed to the fact that the bacterial genomes analyzed are already of high $\mathrm{G}+\mathrm{C}$ percentage, i.e. $62.35 \%$ to $68.49 \%$. Additionally, false positive sRNA candidates obtained during the predictions could also be one of the factors contributing to this observation. The overall size of the sRNA candidates retrieved was not affected by the overall size of the IGRs from where they are predicted (Figure 3C, column graph).

\section{Comparative analysis}

The IGR sequences identified for B. pseudomallei were compared against the 8,920 sRNA candidates using a BLAST-based (blastn) method. The purpose for this comparative analysis is to determine the conservation of sRNA candidates among the closely related bacterial species. As mis-annotations occur in genomes and each of the gene predictors have their own limitations, it was therefore no surprise to detect putative sRNAs from this comparison but not predicted by the sRNA search pipeline. A total of 


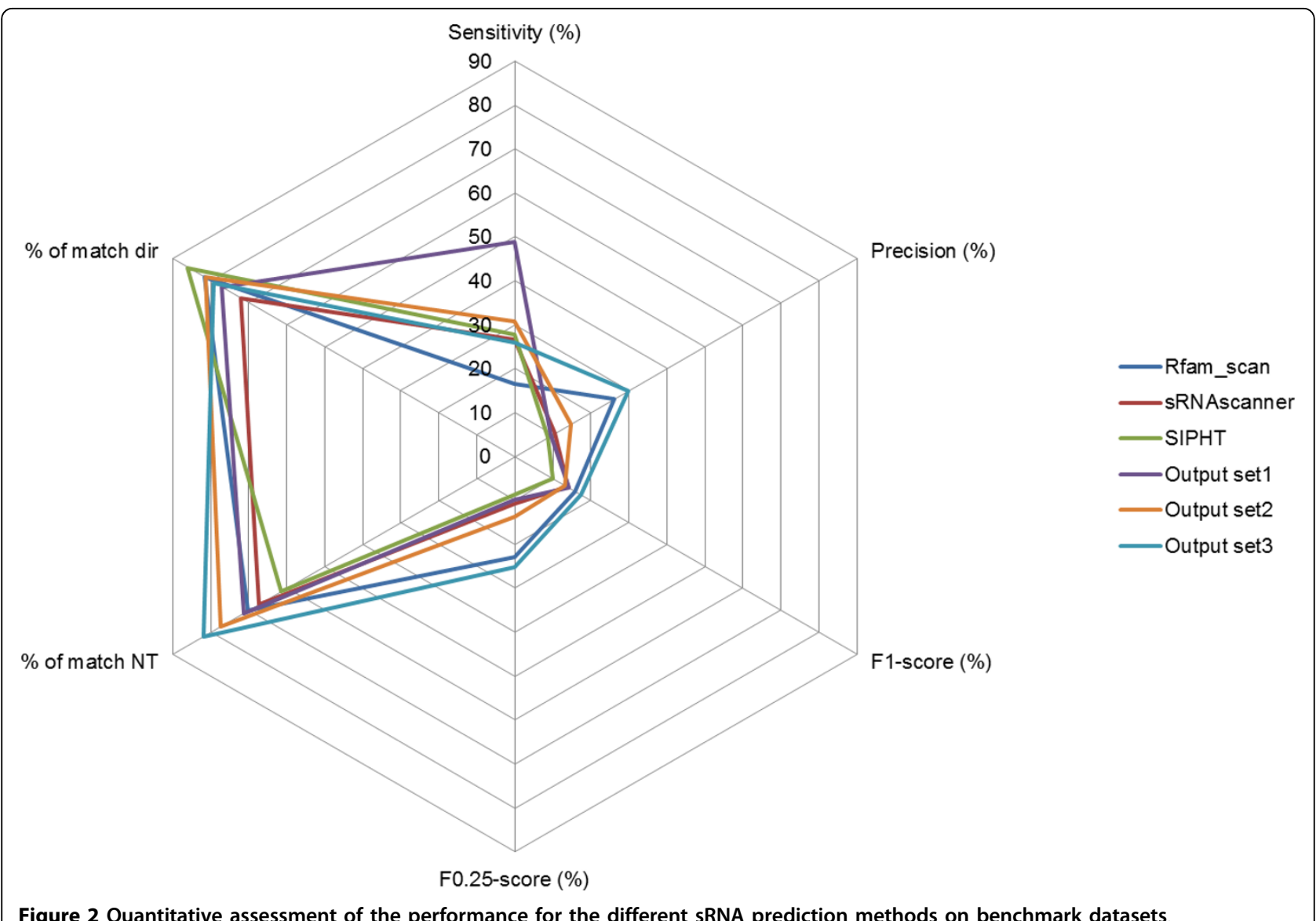

1,213 out of 4,978 (approximately 24\%) B. pseudomallei IGRs were predicted to contain at least one sRNA gene. The complete results list for this comparative analysis is provided as Additional file 4. As two or more sRNA genes could be predicted at the same strand and location, the overlapping candidates were merged before further analysis. For example, if gene A (location: 100 - 200) overlaps with gene B (location 150 - 250), the genes were merged into gene $C$ (location: 100 - 250).

The comparative analysis computationally identified 21 sRNAs in B. pseudomallei that are homologous to previously reported sRNAs (Table 2). These sRNAs include 13 cis-regulatory elements, 6 trans-acting RNAs, 1 ribozyme and 1 sRNA with unknown function. Comparison of the sRNA sequences and predicted secondary structures with entries in Rfam computationally verified the sRNAs predicted (Additional file 5). We cross-referenced the predicted sRNAs with Rfam and found that several of the predicted sRNAs were not noted in the database, while one sRNA recorded in Rfam was missed by our pipeline although the rest were correctly designated (Table 2). The sRNA gene missed during the prediction, LR-PK1 (location: 2314148 - 2314399, reverse strand of chromosome 1), was found to overlap with the infC gene (location: 2314163 - 2314699) located on the same strand of the same chromosome. The gene was not located in the IGRs and therefore it was not predicted in the sRNA gene detection pipeline, which was designed to search for sRNA genes only in the IGRs.

Excluding the 21 homologues to known sRNAs, 20 previously undescribed candidates (also referred to in this paper as novel sRNAs) that were conserved in at least eight out of the fourteen bacterial genomes analyzed were selected for predicted secondary structure comparison where the calculated secondary structures were visually examined. A total of twelve sRNAs with perceivably conserved secondary structures were selected for experimental validation (discussed in the next section).

In order to verify the conservation of the twelve sRNA candidates above within the bacterial kingdom, we generated covariance models for these sRNAs using the sequences from B. pseudomallei, B. mallei, B. thailandensis, B. cenocepacia and $R$. solanacearum and searched against the complete genomic sequences in the RefSeq database using the Infernal program [40] (full results available in Additional file 6). Genes that are unique to B. pseudomallei are of interest because they may help explain the unique features that are not found in other relatives (even the very 


\section{A}

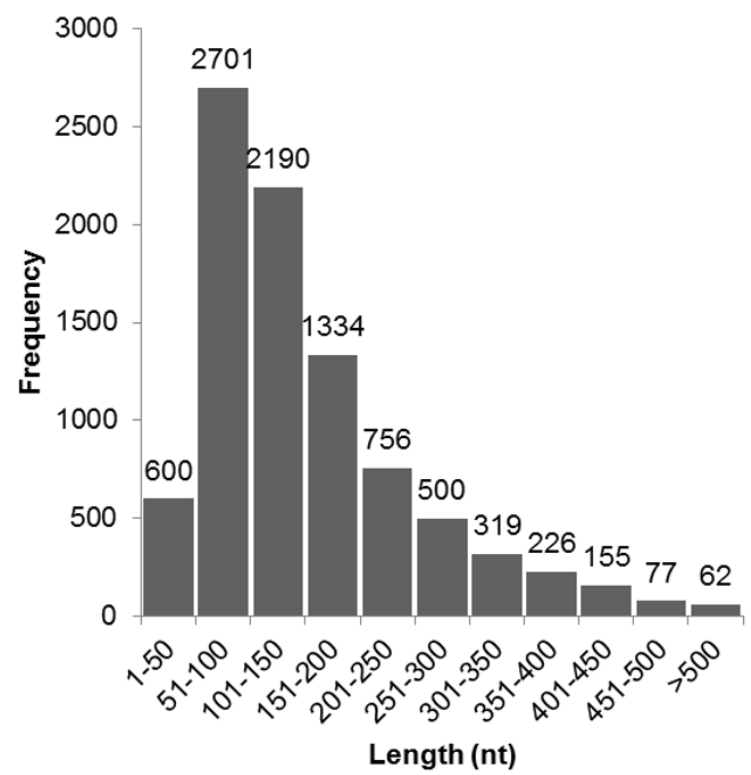

B

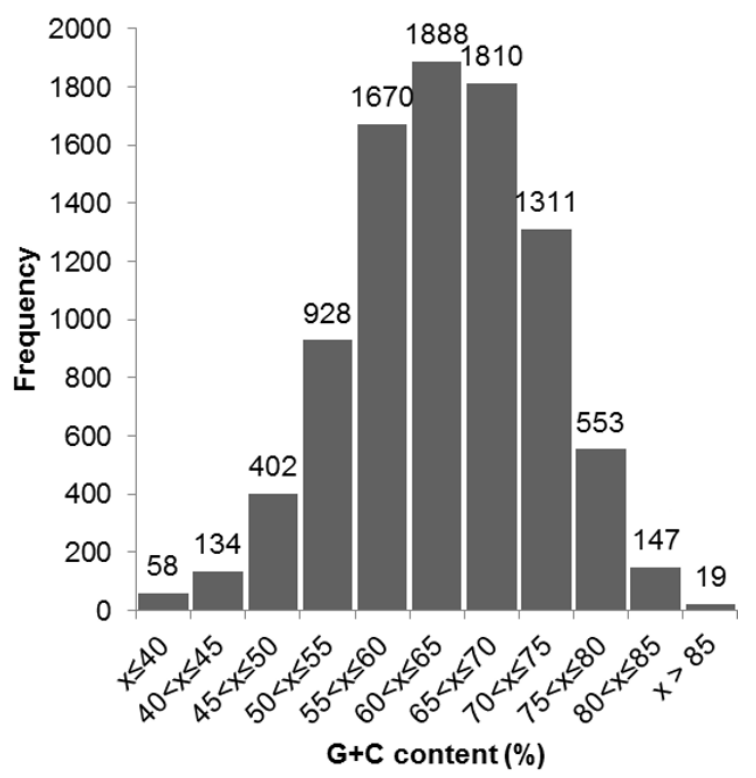

C
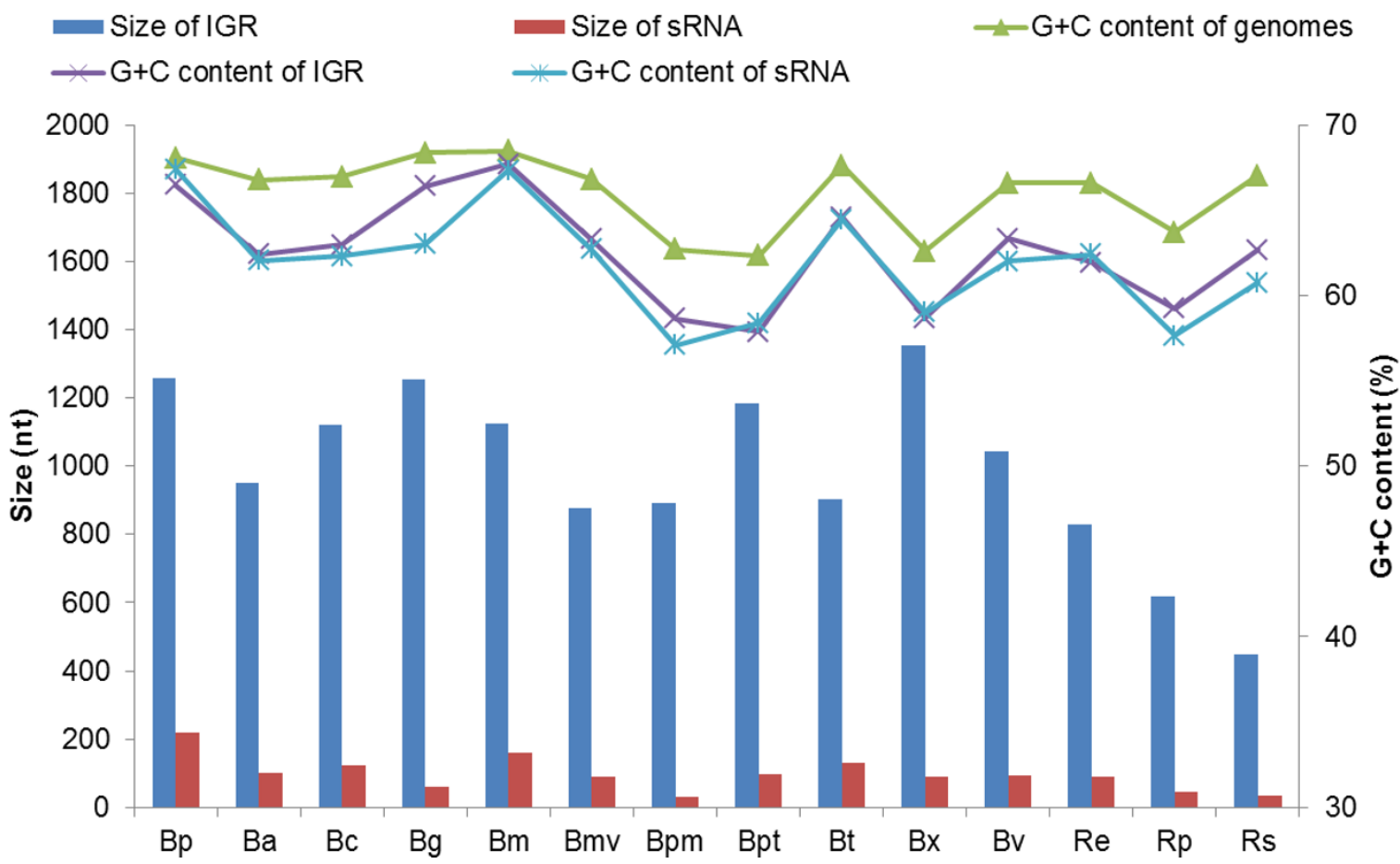

Figure 3 Overview of sRNA candidates. (A) Length distribution. (B) G+C content distribution. (C) Properties of genomes, IGRs and sRNA candidates by species: Column graph - Total IGR and sRNA candidate sizes. Line graph - Overall G+C content of genome, IGR and sRNA candidate sequences. Bp - B. pseudomallei, Ba - B. ambifaria, BC - B. cenocepacia, Bg - B. glumae, Bm - B. mallei, Bmv - B. multivorans, Bpm B. phymatum, Bpt - B. phytofirmans, Bt - B. thailandensis, Bx - B. xenovorans, Bv - B. vietnamiensis, Re - R. eutropha, Rp - R. pickettii, Rs R. solanacearum (further details of the species analysed are available in Additional file 1). 
Table 2 List of B. pseudomallei sRNA sequences with their corresponding sRNA families as reported in Rfam.

\begin{tabular}{|c|c|c|c|c|c|}
\hline No. & sRNA & Chr/strand & Coordinates & Coordinates from Rfam & Type \\
\hline 1. & Cobalamin.1 & $1 /+$ & $1133342 . .1133609$ & (no record) & Riboswitch \\
\hline 2. & Cobalamin. 2 & $1 /+$ & $2072691 . .2072897$ & $2072691 . .2072897$ & Riboswitch \\
\hline 3. & Cobalamin. 3 & $1 /-$ & $2090548 . .2090794$ & (no record) & Riboswitch \\
\hline 4. & Cobalamin. 4 & $1 /+$ & $2090844 . .2091164$ & (no record) & Riboswitch \\
\hline 5. & TPP.1 & $1 /-$ & 1504117..1504234 & $1504117 . .1504234$ & Riboswitch \\
\hline 6. & TPP.2 & $1 /-$ & $3753305 . .3753517$ & 3753386.3753517 & Riboswitch \\
\hline 7. & TPP.3 & $2 /-$ & $1490479 . .1490584$ & $1490479 . .1490584$ & Riboswitch \\
\hline 8. & FMN & $1 /+$ & 772307..772458 & 772307...772458 & Riboswitch \\
\hline 9. & Glycine & $1 /+$ & $3984000 . .3984174$ & 3984000.3984108 & Riboswitch \\
\hline 10. & SAH_riboswitch & $1 /-$ & $3907800 . .3907867$ & 3907800..3907867 & Riboswitch \\
\hline 11. & Mini-ykkC & $1 /+$ & $1359254 . .1359300$ & $1359254 . .1359300$ & Putative riboswitch \\
\hline 12. & sucA & $1 /-$ & $2274625 . .2274707$ & 2274625.2274707 & Putative riboswitch \\
\hline 13. & yybP-ykoY & $1 /+$ & $3066135 . .3066276$ & (no record) & Putative riboswitch \\
\hline 14. & LR-PK1* & $1 /-$ & (not predicted) & $2314148 . .2314399$ & Cis-acting RNA \\
\hline 15. & isrk & $1 /-$ & $98332 . .98411$ & (no record) & Hfq-binding RNA \\
\hline 16. & $6 S$ & $1 /-$ & $1132173 . .1132396$ & 1132214.1132395 & Trans-acting RNA \\
\hline 17. & SRP_bact & $1 /+$ & $1735400 . .1735501$ & $1735400 . .1735501$ & Trans-acting RNA \\
\hline 18. & tmRNA & $1 /+$ & $3041943 . .3042311$ & 3041943.3042311 & Trans-acting RNA \\
\hline 19. & Anti-hemB & $1 /-$ & $3790883 . .3790964$ & (no record) & Trans-acting RNA \\
\hline 20. & CRISPR-DR28 & $1 /+$ & $3578911 . .3578934$ & (no record) & Trans-acting RNA \\
\hline 21. & RNaseP_bact_a & $1 /-$ & $3481314 . .3481770$ & 3481359.3481770 & Ribozyme \\
\hline 22. & P9 & $2 /-$ & $1749221 . .1749373$ & 1749223.1749307 & Gene \\
\hline
\end{tabular}

* The LR-PK1 sRNA was not predicted in this study but has been identified in Rfam.

close ones), in addition to being potential biomarkers for melioidosis. We initially sorted a list of 193 sRNA candidates from B. pseudomallei with no homologues detected during the comparative analysis and searched the 13 genomes of close relatives (all Burkholderia spp. and Ralstonia spp. except B. pseudomallei) by using blastn for similar sequences. The genes with no similar sequences detected were screened again against nine other strains of $B$. pseudomallei (Additional file 1) to confirm their occurrence in all (or most) of the B. pseudomallei strains. Three sRNA candidates were identified from the screening as novel sRNAs unique to $B$. pseudomallei and their transcription under normal growth conditions were tested using RT-PCR (Table 3).

\section{Validation of novel sRNAs using RT-PCR}

A total of fifteen sRNA candidates were selected from the comparative analysis for further verification by RT-PCR of the total RNA extracted from B. pseudomallei D286. Eight candidates were detected in the RT-PCR experiment, each producing PCR products of the expected size with sequences that matched the predictions (Figure 4; Additional file 2). Two candidates, Bp1_Cand449_SIPHT and Bp1_Cand846_SIPHT were tested using the same pair of primers, however subsequent sequencing of the amplified products confirmed only the sequence for Bp1_Cand449_SIPHT.
Analysis of novel sRNAs in Burkholderia pseudomallei

In this section we analyse and discuss several of the novel and validated sRNA genes in our B. pseudomallei D286 model that are the end results of the initial gene prediction and comparative analysis using our pipeline. Bp1_Cand449_SIPHT and Bp1_Cand846_SIPHT, which are highly conserved in Burkholderia, have a $71.7 \%$ sequence identity and highly similar predicted secondary structures (Figure 5A,B). A consensus was found for the two putative sRNAs in the Infernal search (i.e. two distinct Infernal searches for the two sRNAs returned same locations in several genomes, including $R$. solanacearum, Bordetella avium and Janthinobacterium sp. (Figure 5B). From the Infernal search, homologues of this sRNA were detected in bacteria of different order, class and phylum, with one or two copies in each genome. In addition to Burkholderiales, the two sRNAs were also detected in Actinomycetales, Bacillales, Enterobacteriales, Neisseriales, Pseudomonadales as well as Vibrionales. This proposes the possibility that these two sRNA genes are paralogues of each other and not artifacts of a sequence assembly error. As the gene distribution for this putative sRNA covers a wide range of evolutionary distances (Gram positive to Gram negative) and exhibited a low evolutionary rate, it is also quite plausible that Bp1_Cand449_SIPHT and Bp1_Cand846_SIPHT are involved in essential bacterial pathways. The physical 
Table 3 List of RT-PCR validated sRNA genes in conserved in Burkholderia and unique to Burkholderia pseudomallei.

\begin{tabular}{|c|c|c|c|c|}
\hline Name & $\begin{array}{l}\text { Chr/ } \\
\text { Strand }\end{array}$ & Start - end/Length & $\begin{array}{l}\text { GC } \\
\text { content }\end{array}$ & Conservation (Infernal search) \\
\hline \multicolumn{5}{|c|}{ Highly conserved in Burkholderia } \\
\hline Bp1_Cand449_SIPHT* & $1 /-$ & $110185-110354 / 170$ & $50.59 \%$ & Bacteria (detected in Proteobacteria, Bacteroidetes, Firmicutes, etc) \\
\hline Bp1_Cand612_SIPHT & $1 /-$ & $2290411-2290508 / 98$ & $52.04 \%$ & Burkholderia \\
\hline Bp1_Cand684_SIPHT & $1 /-$ & $2768674-2768787 / 114$ & $64.04 \%$ & Bacteria (detected in Actinobacteria, Cyanobacteria, Firmicutes, etc) \\
\hline Bp1_Cand697_SIPHT & $1 /-$ & $2887980-2888055 / 76$ & $64.47 \%$ & Burkholderia \\
\hline Bp1_Cand738_SIPHT & $1 /-$ & $3154052-3154260 / 209$ & $50.72 \%$ & Burkholderia \\
\hline Bp1_Cand871_SIPHT^ & $1 /+$ & $4031759-4031986 / 228$ & $59.21 \%$ & Burkholderia \\
\hline Bp2_Cand287_SIPHT & $2 /-$ & $2326038-2326224 / 187$ & $62.57 \%$ & $\begin{array}{l}\text { Proteobacteria (predominantly in Burkholderiales, detected in } \\
\text { Deltaproteobacteria and Gammaproteobacteria) }\end{array}$ \\
\hline \multicolumn{5}{|l|}{ Unique to $B$. pseudomallei } \\
\hline Bp2_Cand11_sRNAscanner & $2 /-$ & $892370-892562 / 193$ & $36.27 \%$ & B. pseudomallei \\
\hline Bp2_Cand77_SIPHT & $2 /+$ & $575285-575425 / 141$ & $57.45 \%$ & B. pseudomallei \\
\hline
\end{tabular}

* Bp1_Cand449_SIPHT and Bp1_Cand846_SIPHT were verified using the same pair of primers with only Bp1_Cand449_SIPHT also confirmed via sequencing. $\wedge$ Bp1_Cand871_SIPHT was not confirmed via sequencing due to ambiguous sequencing results.

co-localization of Bp1_Cand449_SIPHT, Bp1_Cand846_SIPHT and their homologues in B. thailandensis, R. solanacearum, Bordetella avium and Janthinobacterium sp. were visualized and investigated (Figure 5C). It was found that genes located directly upstream and downstream of the sRNAs were dissimilar in different species. Moreover, the distances between these sRNAs and their flanking genes were also different in each of the genomes. This suggests that these sRNAs are either trans-acting elements or a generic type of cis regulator that can be present in different regulatory pathways.
The homologues of Bp1_Cand287_SIPHT were not only detected in Burkholderiales, but also in $\delta$-proteobacteria and $\gamma$-proteobacteria. From the physical co-localization analysis, the sRNAs were located upstream of genes that are involved in methionine metabolism in most of the genomes (Figure 6A). The sRNA was therefore suggested to be a cis-acting element involved in the modulation of methionine metabolism.

Bp1_Cand684_SIPHT was detected in different groups of bacteria, including Actinobacteria, Cyanobacteria and Firmicutes. Physical co-localization analysis showed that

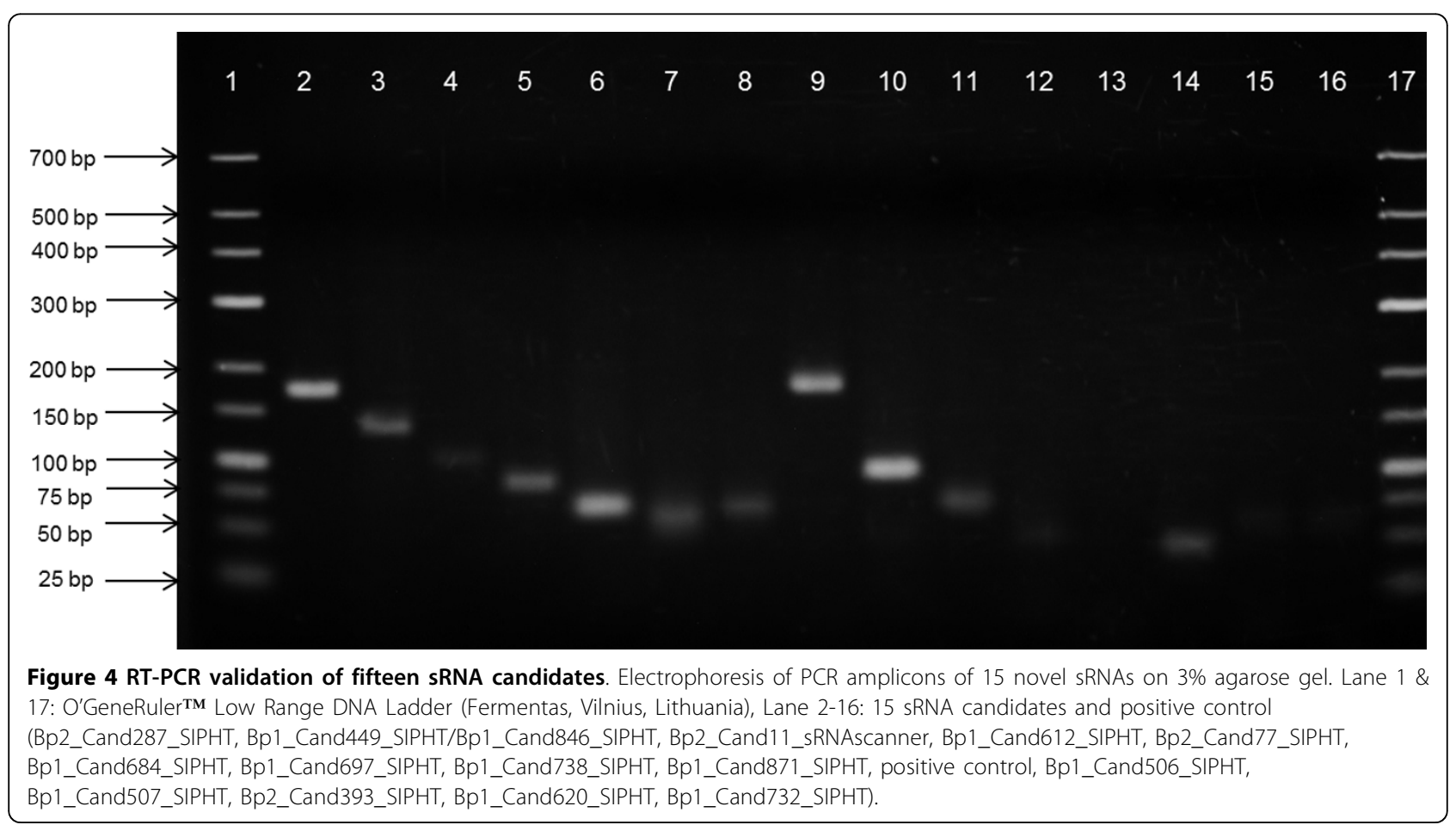




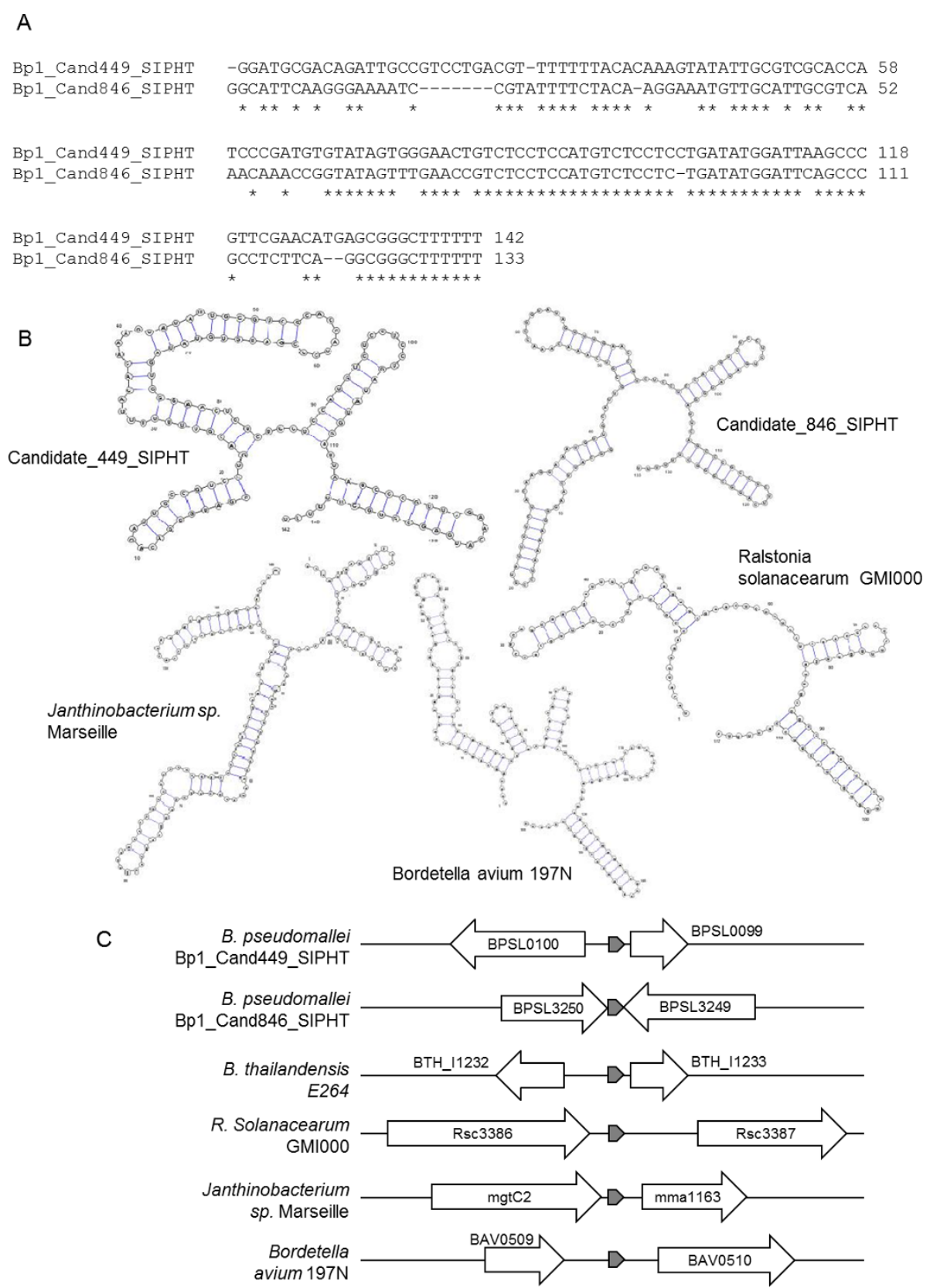

Figure 5 Bp1_Cand449_SIPHT \& Cand846_SIPHT. (A) Sequence alignment of Bp1_Cand449_SIPHT and Bp1_Cand846_SIPHT. (B) Secondary structures of BP1_Cand449_SIPHT, BP1_Cand846_SIPHT and their homologues from Ralstonia solanacearum, Janthinobacterium sp. Marseille and Bordetella avium 197N. (C) Physical co-localization for Bp1_Cand449_SIPHT and Bp1_Cand846_SIPHT and their homologues. The arrows represent the sRNA genes (shaded) and their respective flanking genes. BPSL0100 - O6-methylguanine-DNA methyltransferase, BPSL0099 - glyoxalase/ bleomycin resistance protein/dioxygenase superfamily protein, BPSL3250 - putative LysR-family transcriptional regulator, BPSL3249 - putative outer membrane protein, BTH_I1232 - OsmC/Ohr family protein, BTH_I1233 - ribosomal protein L13, Rsc3386 - outermembrane signal peptide protein, Rsc3387 - Two-component response regulator transcription regulator protein, mgtC2 - Magnesium transporter accessory protein, mma1163 - Transcriptional regulator-like protein, BAV0509 - Hypothetical protein, BAV0510 - O-antigen biosynthesis glucosyltransferase.

the flanking genes were not associated with the same pathways or functions (Figure 6E), suggesting a possible trans-acting role.

Bp1_Cand612_SIPHT, Bp1_Cand697_SIPHT and Bp1_ Cand738_SIPHT are RT-PCR validated sRNA candidates that were found to be Burkholderia-specific. These three sRNAs were not detected in bacteria other than Burkholderia spp. during the Infernal search. From the physical co-localization analysis, each of these three sRNA genes has similar flanking genes in different Burkholderia spp. 


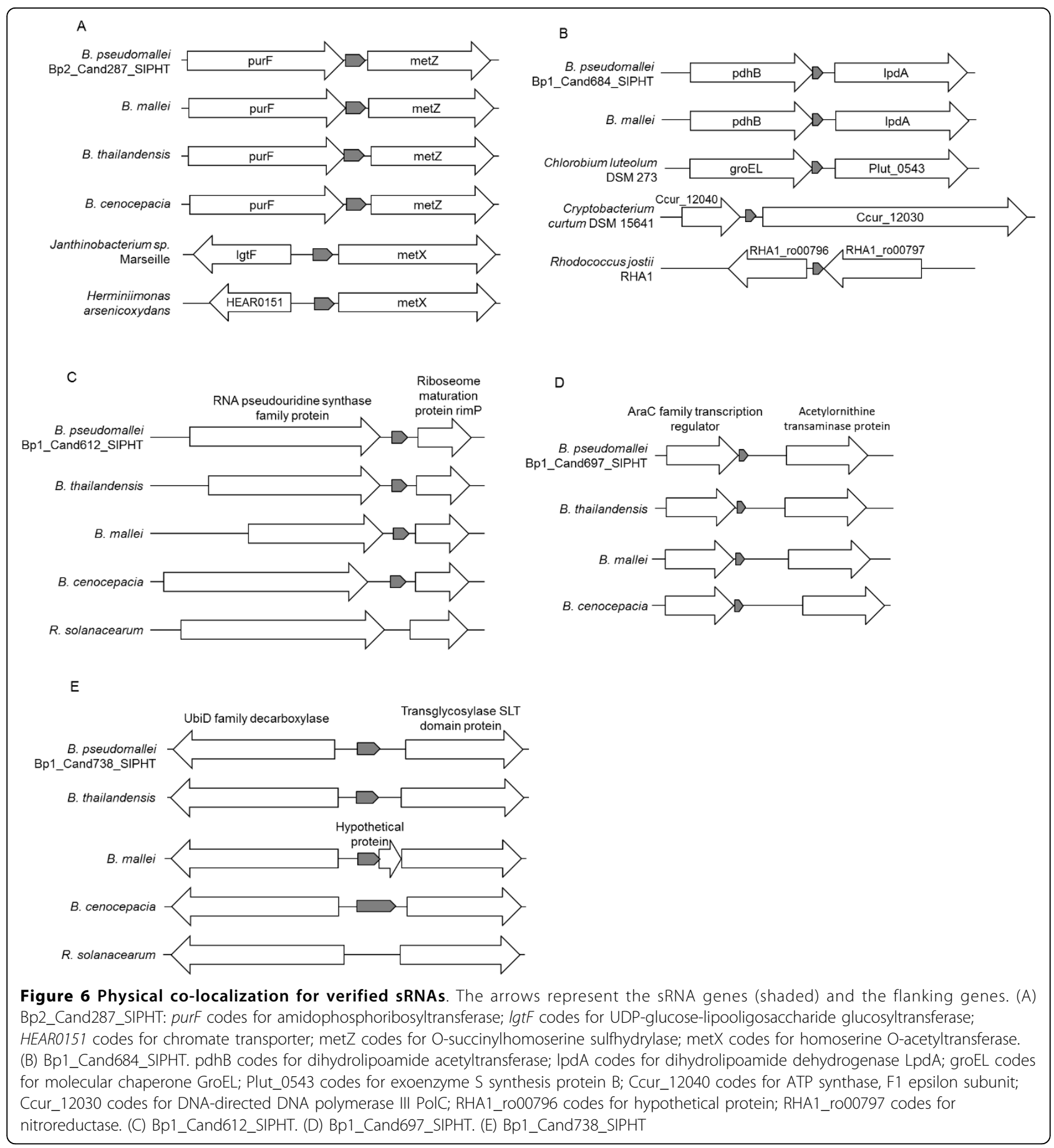

(Figure 6B-D). For Bp1_Cand612_SIPHT and Bp1_ Cand697_SIPHT, although $R$. solanacearum has a similar gene arrangement at the equivalent regions, no such sRNA genes were predicted in that genome.

In prokaryotes, $\mathrm{Hfq}$ proteins regulate translation by modulating the structure of numerous RNA molecules. The motif 5'-AAYAAYAA-3' is enriched in Hfq-binding RNAs and binding to Hfq was confirmed by DMS footprinting [60] while other researchers have shown that Hfq binds sRNAs with a preference for AU-rich sequences [61-63]. One of the identified B. pseudomallei-specific sRNAs, Bp2_Cand11_sRNAscanner, was found to contain a putative Hfq-binding motif (5'-AAYAAYAA-3') and several AU-rich regions. The secondary structures of the sRNAs showed that the motif and one of the AU-rich region were accessible (i.e. located at the loop region), 


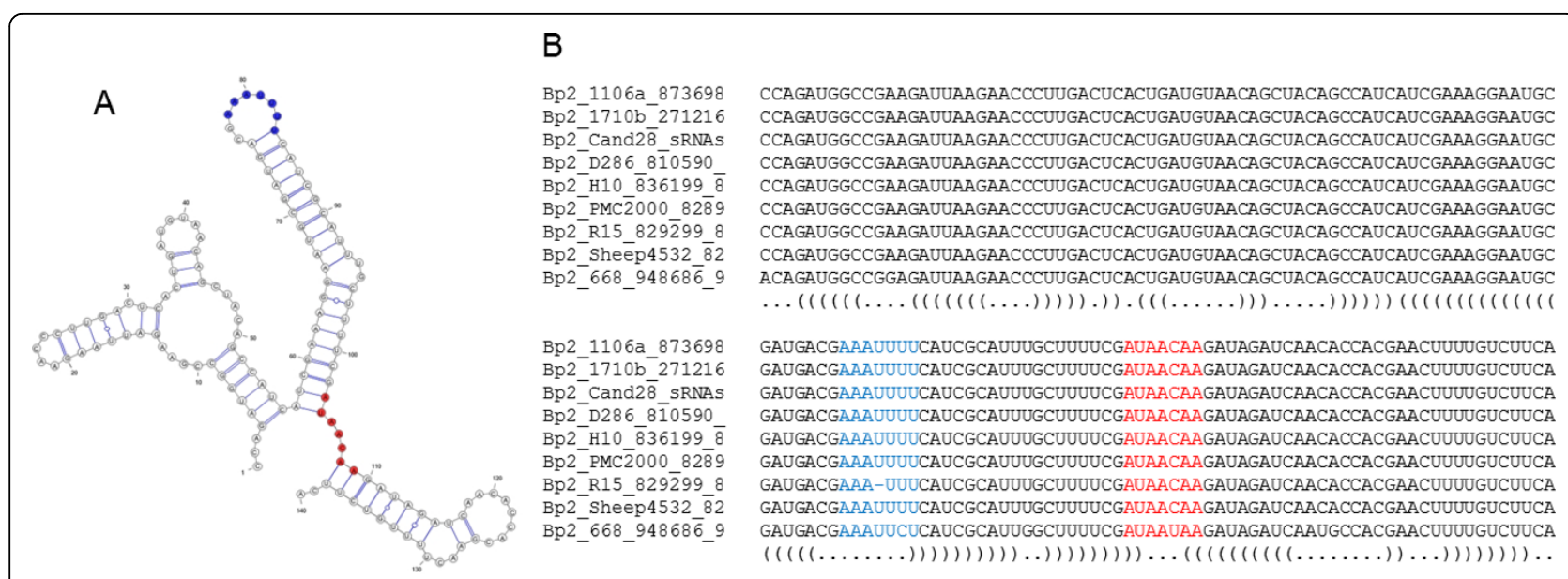

Figure 7 Bp2_Cand11_sRNAscanner. Consensus secondary structure predicted by RNAalifold (A) using the annotated alignment generated by LOCARNA as shown in (B). The highlighted region in the alignment are putative Hfq-binding motifs.

implying that Bp2_Cand11_sRNAscanner could be a Hfqdependent RNA (Figure 7).

\section{Conclusions}

A total of 1,306 B. pseudomallei sRNA genes were predicted in this study of which: 21 have homologs in Rfam; 15 novel sRNAs were shortlisted due to their conservation in Burkholderia spp. or different B. pseudomallei strains; and 8 of these were verified experimentally. Though the functions for the novel sRNAs obtained in this study remain unknown, their presence in B. pseudomallei is evidence that sRNAs are indeed involved in this bacterium's many different cellular activities that may include regulation of pathogenesis and virulence mechanisms as well as adaptation to environmentally induced changes.

\section{Additional material}

Additional file 1: Bacterial genomes studied. List of genome

sequences used for sRNA prediction and analysis.

Additional file 2: Primers. Nucleotide sequences of PCR primers for amplifying sRNA genes.

Additional file 3: sRNA candidates. List of SRNA candidates predicted in fourteen bacterial genomes.

Additional file 4: Comparative analysis. The results of comparative analysis of sRNA candidates predicted.

Additional file 5: Secondary structures of known sRNAs identified Secondary structures visualization for known sRNAs discovered in this study and reference structures predicted from Rfam entries.

Additional file 6: Infernal search results. The results of putative sRNA homologues search using Infernal.

\section{Acknowledgements}

Research funding was provided by Universiti Kebangsaan Malaysia via the UKM research university grants UKM-GUP-KPB-08-33-132 and DIP-2012-13 and the Ministry of Higher Education Malaysia grant ERGS/1/2012/STG08/ UKM/02/5. KJS was funded by a National Science Fellowship from the
Ministry of Science, Technology and Innovation, Malaysia. CSF was funded by the MyMaster-MyBrain 15 scholarship from the Ministry of Higher Education, Malaysia and the Universiti Kebangsaan Malaysia Zamalah postgraduate research fellowship.

This article has been published as part of BMC Genomics Volume 13 Supplement 7, 2012: Eleventh International Conference on Bioinformatics (InCoB2012): Computational Biology. The full contents of the supplement are available online at http://www.biomedcentral.com/bmcgenomics/ supplements/13/S7.

\section{Author details}

${ }^{1}$ School of Biosciences and Biotechnology, Faculty of Science and Technology, Universiti Kebangsaan Malaysia, 43600 UKM Bangi, Malaysia. ${ }^{2}$ Codon Genomics SB, Jalan Bandar 18, Pusat Bandar Puchong, Selangor Darul Ehsan, Malaysia. ${ }^{3}$ Malaysia Genome Institute, Jalan Bangi Lama, 43000 Kajang, Malaysia.

\section{Authors' contributions}

KUS developed the SRNA prediction pipeline and carried out the computational sRNA discovery and comparative analyses. SN contributed to the genome sequence analysis of the B. pseudomallei strains. CSF carried out the validation experiments. KJS, CSF and MFR wrote the manuscript. RM contributed to the design and execution of the validation experiments. SN and MFR revised the manuscript. All authors submitted comments, read and approved the final manuscript.

\section{Competing interests}

The authors declare that they have no competing interests.

Published: 13 December 2012

\section{References}

1. Argaman L, Hershberg R, Vogel J, Bejerano G, Wagner EG, Margalit H, Altuvia $S$ : Novel small RNA-encoding genes in the intergenic regions of Escherichia coli. Curr Biol 2001, 11:941-950.

2. Saito $S$, Kakeshita $H$, Nakamura $K$ : Novel small RNA-encoding genes in the intergenic regions of Bacillus subtilis. Gene 2009, 428:2-8.

3. Fedor MJ, Williamson JR: The catalytic diversity of RNAs. Nat Rev Mol Cell Biol 2005, 6:399-412

4. Wu Q, Huang L, Zhang Y: The structure and function of catalytic RNAs. Sci China C Life Sci 2009, 52:232-244.

5. Beisel $\mathrm{CL}$, Storz G: Base pairing small RNAs and their roles in global regulatory networks. FEMS Microbiol Rev 2010, 34:866-882.

6. Storz G, Opdyke JA, Zhang A: Controlling mRNA stability and translation with small, noncoding RNAs. Curr Opin Microbiol 2004, 7:140-144.

7. Storz G, Vogel J, Wassarman KM: Regulation by small RNAs in bacteria: expanding frontiers. Mol Cell 2011, 43:880-891. 
8. Hershberg R, Altuvia $\mathrm{S}$, Margalit H: A survey of small RNA-encoding genes in Escherichia coli. Nucleic Acids Res 2003, 31:1813-1820.

9. Gardner PP, Daub J, Tate JG, Nawrocki EP, Kolbe DL, Lindgreen S, Wilkinson AC, Finn RD, Griffiths-Jones S, Eddy SR, Bateman A: Rfam: updates to the RNA families database. Nucleic Acids Res 2009, 37:D136-140.

10. Huang HY, Chang HY, Chou CH, Tseng CP, Ho SY, Yang CD, Ju YW, Huang HD: sRNAMap: genomic maps for small non-coding RNAs, their regulators and their targets in microbial genomes. Nucleic Acids Res 2009, 37:D150-154.

11. Vogel J, Papenfort K: Small non-coding RNAs and the bacterial outer membrane. Current Opinion in Microbiology 2006, 9:605-611.

12. Benito Y, Kolb FA, Romby P, Lina G, Etienne J, Vandenesch F: Probing the structure of RNAIll, the Staphylococcus aureus agr regulatory RNA, and identification of the RNA domain involved in repression of protein A expression. RNA 2000, 6:668-679.

13. Altuvia S, Zhang A, Argaman L, Tiwari A, Storz G: The Escherichia coli OxyS regulatory RNA represses fhIA translation by blocking ribosome binding. EMBO J 1998, 17:6069-6075.

14. Lenz DH, Mok KC, Lilley BN, Kulkarni RV, Wingreen NS, Bassler BL: The small RNA chaperone Hfq and multiple small RNAs control quorum sensing in Vibrio harveyi and Vibrio cholerae. Cell 2004, 118:69-82.

15. Weilbacher T, Suzuki K, Dubey AK, Wang X, Gudapaty S, Morozov I, Baker CS, Georgellis D, Babitzke P, Romeo T: A novel sRNA component of the carbon storage regulatory system of Escherichia coli. Mol Microbiol 2003, 48:657-670.

16. Barrick JE, Sudarsan N, Weinberg Z, Ruzzo WL, Breaker RR: 6S RNA is a widespread regulator of eubacterial RNA polymerase that resembles an open promoter. RNA 2005, 11:774-784.

17. Chabelskaya S, Gaillot O, Felden B: A Staphylococcus aureus small RNA is required for bacterial virulence and regulates the expression of an immune-evasion molecule. PLoS Pathog 2010, 6:e1000927.

18. Padalon-Brauch G, Hershberg R, Elgrably-Weiss M, Baruch K, Rosenshine I, Margalit H, Altuvia S: Small RNAs encoded within genetic islands of Salmonella typhimurium show host-induced expression and role in virulence. Nucleic Acids Res 2008, 36:1913-1927.

19. Muers M: Small RNAs: microbial metatranscriptomics goes deep. Nat Rev Genet 2009, 10:426-427.

20. Song T, Wai SN: A novel sRNA that modulates virulence and environmental fitness of Vibrio cholerae. RNA Biol 2009, 6:254-258.

21. Camacho EM, Serna A, Madrid C, Marques S, Fernandez R, de la Cruz F, Juarez A, Casadesus J: Regulation of finP transcription by DNA adenine methylation in the virulence plasmid of Salmonella enterica. J Bacteriol 2005, 187:5691-5699.

22. Giangrossi M, Prosseda G, Tran CN, Brandi A, Colonna B, Falconi M: A novel antisense RNA regulates at transcriptional level the virulence gene ics $A$ of Shigella flexneri. Nucleic Acids Res 2010, 38:3362-3375.

23. Robertson GT, Roop RM Jr: The Brucella abortus host factor I (HF-I) protein contributes to stress resistance during stationary phase and is a major determinant of virulence in mice. Mol Microbiol 1999, 34:690-700.

24. Brown $\mathrm{L}$, Elliott $\mathrm{T}$ : Efficient translation of the RpoS sigma factor in Salmonella typhimurium requires host factor $\mathrm{I}$, an RNA-binding protein encoded by the hfa gene. J Bacteriol 1996, 178:3763-3770.

25. Ding $Y$, Davis BM, Waldor MK: Hfq is essential for Vibrio cholerae virulence and downregulates sigma expression. Mol Microbiol 2004, 53:345-354.

26. Nakao H, Watanabe H, Nakayama S, Takeda T: yst gene expression in Yersinia enterocolitica is positively regulated by a chromosomal region that is highly homologous to Escherichia coli host factor 1 gene ( $h f q$ ). Mol Microbiol 1995, 18:859-865.

27. Sonnleitner E, Hagens S, Rosenau F, Wilhelm S, Habel A, Jager KE, Blasi U: Reduced virulence of a hfq mutant of Pseudomonas aeruginosa 01 Microb Pathog 2003, 35:217-228.

28. Zhang A, Wassarman KM, Rosenow C, Tjaden BC, Storz G, Gottesman S: Global analysis of small RNA and mRNA targets of Hfq. Mol Microbiol 2003, 50:1111-1124.

29. Gan YH: Interaction between Burkholderia pseudomallei and the host immune response: sleeping with the enemy? I Infect Dis 2005, 192:1845-1850.

30. Pitt TL, Trakulsomboon S, Dance DA: Molecular phylogeny of Burkholderia pseudomallei. Acta Trop 2000, 74:181-185.

31. Holden MT, Titball RW, Peacock SJ, Cerdeno-Tarraga AM, Atkins T, Crossman LC, Pitt T, Churcher C, Mungall K, Bentley SD, et al: Genomic plasticity of the causative agent of melioidosis, Burkholderia pseudomallei. Proc Natl Acad Sci USA 2004, 101:14240-14245.

32. Hasselbring BM, Patel MK, Schell MA: Dictyostelium discoideum as a model system for identification of Burkholderia pseudomallei virulence factors. Infect Immun 2011, 79:2079-2088.

33. O'Quinn AL, Wiegand EM, Jeddeloh JA: Burkholderia pseudomallei kills the nematode Caenorhabditis elegans using an endotoxin-mediated paralysis. Cell Microbiol 2001, 3:381-393.

34. Huang CT: What is Pseudomonas pseudomallei. Elixir 1976, 70-72.

35. Choy JL, Mayo M, Janmaat A, Currie BJ: Animal melioidosis in Australia. Acta Tropica 2000, 74:153-158.

36. Ngauy V, Lemeshev Y, Sadkowski L, Crawford G: Cutaneous melioidosis in a man who was taken as a prisoner of war by the Japanese during World War II. J Clin Microbiol 2005, 43:970-972.

37. Pumpuang A, Chantratita N, Wikraiphat C, Saiprom N, Day NP, Peacock SJ, Wuthiekanun V: Survival of Burkholderia pseudomallei in distilled water for 16 years. Trans R Soc Trop Med Hyg 2011, 105:598-600.

38. Cruz-Migoni A, Hautbergue GM, Artymiuk PJ, Baker PJ, Bokori-Brown M, Chang C-T, Dickman MJ, Essex-Lopresti A, Harding SV, Mahadi NM, et al: A Burkholderia pseudomallei toxin inhibits helicase activity of translation factor elF4A. Science 2011, 334:821-824.

39. Coenye T, Drevinek P, Mahenthiralingam E, Shah SA, Gill RT, Vandamme P, Ussery DW: Identification of putative noncoding RNA genes in the Burkholderia cenocepacia J2315 genome. FEMS Microbiol Lett 2007, 276:83-92.

40. Nawrocki EP, Kolbe DL, Eddy SR: Infernal 1.0: inference of RNA alignments. Bioinformatics 2009, 25:1335-1337.

41. Rivas E, Eddy SR: Noncoding RNA gene detection using comparative sequence analysis. BMC Bioinformatics 2001, 2:8

42. Gruber AR, Findeiss S, Washietl S, Hofacker IL, Stadler PF: Rnaz 2.0: improved noncoding RNA detection. Pac Symp Biocomput 2010, 15:69-79.

43. Washietl S, Hofacker IL, Stadler PF: Fast and reliable prediction of noncoding RNAs. Proc Natl Acad Sci USA 2005, 102:2454-2459.

44. Livny J, Brencic A, Lory S, Waldor MK: Identification of 17 Pseudomonas aeruginosa sRNAs and prediction of sRNA-encoding genes in 10 diverse pathogens using the bioinformatic tool sRNAPredict2. Nucleic Acids Res 2006, 34:3484-3493.

45. Livny J, Fogel MA, Davis BM, Waldor MK: sRNAPredict: an integrative computational approach to identify sRNAs in bacterial genomes. Nucleic Acids Res 2005, 33:4096-4105.

46. Sridhar J, Narmada SR, Sabarinathan R, Ou H-Y, Deng Z, Sekar K, Rafi ZA, Rajakumar K: sRNAscanner: a computational tool for intergenic small RNA detection in bacterial genomes. PLoS One 2010, 5:e11970.

47. Herbig A, Nieselt K: nocoRNAc: characterization of non-coding RNAs in prokaryotes. BMC Bioinformatics 2011, 12:40.

48. Livny J, Teonadi H, Livny M, Waldor MK: High-throughput, kingdom-wide prediction and annotation of bacterial non-coding RNAs. PLOS One 2008, 3:e3197.

49. Rutherford K, Parkhill J, Crook J, Horsnell T, Rice P, Rajandream MA, Barrell B: Artemis: sequence visualization and annotation. Bioinformatics 2000 16:944-945.

50. Altschul SF, Madden TL, Schaffer AA, Zhang J, Zhang Z, Miller W, Lipman DJ: Gapped BLAST and PSI-BLAST: a new generation of protein database search programs. Nucleic Acids Res 1997, 25:3389-3402.

51. Carver TJ, Rutherford KM, Berriman M, Rajandream MA, Barrell BG, Parkhill J: ACT: the Artemis Comparison Tool. Bioinformatics 2005, 21:3422-3423.

52. Zuker M: Mfold web server for nucleic acid folding and hybridization prediction. Nucleic Acids Res 2003, 31:3406-3415.

53. Gruber AR, Lorenz R, Bernhart SH, Neubock R, Hofacker IL: The Vienna RNA websuite. Nucleic Acids Res 2008, 36:W70-74

54. Darty K, Denise A, Ponty Y: VARNA: interactive drawing and editing of the RNA secondary structure. Bioinformatics 2009, 25:1974-1975.

55. Smith C, Heyne S, Richter AS, Will S, Backofen R: Freiburg RNA Tools: a web server integrating INTARNA, EXPARNA and LOCARNA. Nucleic Acids Res 2010, 38(Suppl):W373-377.

56. Lee SH, Chong CE, Lim BS, Chai SJ, Sam KK, Mohamed R, Nathan S: Burkholderia pseudomallei animal and human isolates from Malaysia exhibit different phenotypic characteristics. Diagn Microbiol Infect Dis 2007, 58:263-270.

57. Lu X, Goodrich-Blair H, Tjaden B: Assessing computational tools for the discovery of small RNA genes in bacteria. RNA 2011, 17:1635-1647. 
58. van Rijsbergen CJ: Information Retrieval Butterworths, London; 1979.

59. Schattner P: Searching for RNA genes using base-composition statistics. Nucleic Acids Res 2002, 30:2076-2082.

60. Lorenz C, Gesell T, Zimmermann B, Schoeberl U, Bilusic I, Rajkowitsch L, Waldsich C, von Haeseler A, Schroeder R: Genomic SELEX for Hfq-binding RNAs identifies genomic aptamers predominantly in antisense transcripts. Nucleic Acids Res 2010, 38:3794-3808.

61. Brennan RG, Link TM: Hfq structure, function and ligand binding. Curr Opin Microbiol 2007, 10:125-133.

62. Valentin-Hansen P, Eriksen M, Udesen C: The bacterial Sm-like protein Hfq: a key player in RNA transactions. Mol Microbiol 2004, 51:1525-1533.

63. Otaka H, Ishikawa H, Morita T, Aiba H: PolyU tail of rho-independent terminator of bacterial small RNAs is essential for $\mathrm{Hfq}$ action. Proc Natl Acad Sci USA 2011, 108:13059-13064.

64. Rivas E, Klein RJ, Jones TA, Eddy SR: Computational identification of noncoding RNAs in E. coli by comparative genomics. Curr Biol 2001, 11:1369-1373.

65. Chen S, Lesnik EA, Hall TA, Sampath R, Griffey RH, Ecker DJ, Blyn LB: A bioinformatics based approach to discover small RNA genes in the Escherichia coli genome. Biosystems 2002, 65:157-177.

66. Panek J, Bobek J, Mikulik K, Basler M, Vohradsky J: Biocomputational prediction of small non-coding RNAs in Streptomyces. BMC Genomics 2008, 9:217.

67. Voss B, Georg J, Schon V, Ude S, Hess WR: Biocomputational prediction of non-coding RNAs in model cyanobacteria. BMC Genomics 2009, 10:123.

68. Geissmann T, Chevalier C, Cros MJ, Boisset S, Fechter P, Noirot C, Schrenzel J, Francois P, Vandenesch F, Gaspin C, Romby P: A search for small noncoding RNAs in Staphylococcus aureus reveals a conserved sequence motif for regulation. Nucleic Acids Res 2009, 37:7239-7257.

69. Tran TT, Zhou F, Marshburn S, Stead M, Kushner SR, Xu Y: De novo computational prediction of non-coding RNA genes in prokaryotic genomes. Bioinformatics 2009, 25:2897-2905.

doi:10.1186/1471-2164-13-S7-S13

Cite this article as: Khoo et al:: Computational discovery and RT-PCR validation of novel Burkholderia conserved and Burkholderia pseudomallei unique sRNAs. BMC Genomics 2012 13(Suppl 7):S13.

\section{Submit your next manuscript to BioMed Central and take full advantage of:}

- Convenient online submission

- Thorough peer review

- No space constraints or color figure charges

- Immediate publication on acceptance

- Inclusion in PubMed, CAS, Scopus and Google Scholar

- Research which is freely available for redistribution

Submit your manuscript at www.biomedcentral.com/submit
Biomed Central 